\title{
Faithfulness of a functor of Quillen
}

\author{
WILLIAM G DWYER \\ ANDREI RĂDULESCU-BANU \\ SEBASTIAN THOMAS
}

\begin{abstract}
There exists a canonical functor from the category of fibrant objects of a model category modulo cylinder homotopy to its homotopy category. We show that this functor is faithful under certain conditions, but not in general.
\end{abstract}

$18 \mathrm{G} 55,55 \mathrm{U} 35$

\section{Introduction}

We let $\mathcal{M}$ be a model category. Quillen defines in [5, Chapter I, Section 1] a homotopy relation on the full subcategory $\mathbf{F i b}(\mathcal{M})$ of fibrant objects, using cylinders. He obtains a quotient category $\operatorname{Fib}(\mathcal{M}) / \stackrel{\mathrm{c}}{\sim}$ and a canonical functor

$$
\operatorname{Fib}(\mathcal{M}) / \stackrel{\mathfrak{c}}{\sim} \rightarrow \operatorname{Ho} \operatorname{Fib}(\mathcal{M}) .
$$

The question occurs whether this functor is faithful.

We show that it is faithful if $\mathcal{M}$ is left proper and fulfills an additional technical condition. Moreover, we show by an example that it is not faithful in general.

\section{Conventions and notation}

- The composite of morphisms $f: X \rightarrow Y$ and $g: Y \rightarrow Z$ is denoted by $f g: X \rightarrow Z$.

- Given $n \in \mathbb{N}_{0}$, we abbreviate $\mathbb{Z} / n:=\mathbb{Z} / n \mathbb{Z}$. Given $k, m, n \in \mathbb{N}_{0}$, we write $k: \mathbb{Z} / m \rightarrow \mathbb{Z} / n, a+m \mathbb{Z} \mapsto k a+n \mathbb{Z}$, provided $n$ divides $k m$.

- Given a category $\mathcal{C}$ with finite coproducts and objects $X, Y \in \mathrm{Ob} \mathcal{C}$, we denote by $X \amalg Y$ a (chosen) coproduct. The embedding $X \rightarrow X \amalg Y$ is denoted by emb $_{0}$, the embedding $Y \rightarrow X \amalg Y$ by emb e $_{1}$ Given morphisms $f: X \rightarrow Z$ and $g: Y \rightarrow Z$ in $\mathcal{C}$, the induced morphism $X \amalg Y \rightarrow Z$ is denoted by $\left(\begin{array}{l}f \\ g\end{array}\right)$.

- Given a category $\mathcal{C}$ and an object $X \in \mathrm{Ob} \mathcal{C}$, the category of objects in $\mathcal{C}$ under $X$ will be denoted by $(X \downarrow \mathcal{C})$. The objects in $(X \downarrow \mathcal{C})$ are denoted by $(Y, f)$, where $Y \in \operatorname{Ob} \mathcal{C}$ and $f: X \rightarrow Y$ is a morphism in $\mathcal{C}$. 


\section{Preliminaries from homotopical algebra}

We recall some basic facts from homotopical algebra. Our main reference is Quillen [5, Chapter I, Section 1].

\section{Model categories}

Throughout this note, we let $\mathcal{M}$ be a model category [5, Chapter I, Section 1, Definition 1]. In $\mathcal{M}$, there are three kinds of distinguished morphisms, called cofibrations, fibrations and weak equivalences. Cofibrations are closed under pushouts. If weak equivalences in $\mathcal{M}$ are closed under pushouts along cofibrations, $\mathcal{M}$ is said to be left proper [3, Definition 13.1.1(1)].

An object $X \in \mathrm{Ob} \mathcal{M}$ is said to be fibrant if the unique morphism $\mathcal{M} \rightarrow *$ is a fibration, where $*$ is a (chosen) terminal object in $\mathcal{M}$. The full subcategory of $\mathcal{M}$ of fibrant objects is denoted by $\operatorname{Fib}(\mathcal{M})$.

The homotopy category of $\mathcal{C}=\mathcal{M}$ resp. $\mathcal{C}=\operatorname{Fib}(\mathcal{M})$ is a localisation of $\mathcal{C}$ with respect to the weak equivalences in $\mathcal{C}$ and is denoted by $\operatorname{Ho} \mathcal{C}$. The localisation functor of $\mathrm{Ho} \mathcal{C}$ is denoted by $\Gamma=\Gamma^{\mathrm{Ho}} \mathcal{C}: \mathcal{C} \rightarrow \mathrm{Ho} \mathcal{C}$.

Given an object $X \in \mathrm{Ob} \mathcal{M}$, the category $(X \downarrow \mathcal{M})$ of objects under $X$ obtains a model category structure where a morphism in $(X \downarrow \mathcal{M})$ is a weak equivalence resp. a cofibration resp. a fibration if and only if it is one in $\mathcal{M}$.

\section{Homotopies}

A cylinder for an object $X \in \mathrm{Ob} \mathcal{M}$ consists of an object $Z \in \mathrm{Ob} \mathcal{M}$, a cofibration $\left(\begin{array}{l}\text { inso } \\ \text { ins } 1\end{array}\right)=$ ins $=$ ins $^{Z}: X \amalg X \rightarrow Z$ and a weak equivalence $\mathrm{s}=\mathrm{s}^{Z}: Z \rightarrow X$ such that ins $\mathrm{s}=\left(\begin{array}{l}1 \\ 1\end{array}\right)$.

Given parallel morphisms $f, g: X \rightarrow Y$ in $\mathcal{M}$, we say that $f$ is cylinder homotopic to $g$, written $f \stackrel{\mathrm{c}}{\sim} g$, if there exists a cylinder $Z$ for $X$ and a morphism $H: Z \rightarrow Y$ with ins ${ }_{0} H=f$ and ins ${ }_{1} H=g$. In this case, $H$ is said to be a cylinder homotopy from $f$ to $g$. (In the literature, cylinder homotopy is also called left homotopy; see Quillen [5, Chapter I, Section 1, Definitions 3-4, Lemma 1].) The relation $\stackrel{c}{\sim}$ is reflexive and symmetric, but in general not transitive. Moreover, $\stackrel{c}{\sim}$ is compatible with composition in $\operatorname{Fib}(\mathcal{M})$. We denote by $\operatorname{Fib}(\mathcal{M}) / \stackrel{\text { c }}{\sim}$ the quotient category of $\operatorname{Fib}(\mathcal{M})$ with respect to the congruence generated by $\stackrel{\mathrm{c}}{\sim}$. 


\section{Quillen's homotopy category theorem}

There are dual notions to fibrant objects, cylinders, cylinder homotopic $\stackrel{\mathrm{c}}{\sim}$, the full subcategory of fibrant objects $\operatorname{Fib}(\mathcal{M})$, its quotient category $\operatorname{Fib}(\mathcal{M}) / \stackrel{\mathcal{c}}{\sim}$ and its homotopy category $\operatorname{Ho} \mathbf{F i b}(\mathcal{M})$, namely cofibrant objects, path objects, path homotopic $\stackrel{\mathrm{p}}{\sim}$, the full subcategory of cofibrant objects $\operatorname{Cof}(\mathcal{M})$, its quotient category $\operatorname{Cof}(\mathcal{M}) / \stackrel{\text { p }}{\sim}$ and its homotopy category $\operatorname{Ho} \operatorname{Cof}(\mathcal{M})$, respectively. Moreover, an object $X \in \mathrm{Ob} \mathcal{M}$ is said to be bifibrant if it is cofibrant and fibrant. On the full subcategory of bifibrant objects $\operatorname{Bif}(\mathcal{M})$, the relations $\stackrel{\mathrm{c}}{\sim}$ and $\stackrel{\mathrm{p}}{\sim}$ coincide and yield a congruence. One writes $\sim:=\stackrel{\mathrm{c}}{\sim}=\stackrel{\mathrm{p}}{\sim}$ in this case, and the quotient category is denoted by $\operatorname{Bif}(\mathcal{M}) / \sim$. Moreover, $\mathrm{Ho} \operatorname{Bif}(\mathcal{M})$ is a localisation of $\operatorname{Bif}(\mathcal{M})$ with respect to the weak equivalences in $\operatorname{Bif}(\mathcal{M})$.

Quillen's homotopy category theorem [5, Chapter I, Section 1, Theorem 1] (cf Hovey [4, Corollary 1.2.9, Theorem 1.2.10]) states that the various inclusion and localisation functors induce the following commutative diagram, where the functors labeled by $\simeq$ are equivalences and the functor labeled by $\cong$ is an isofunctor.

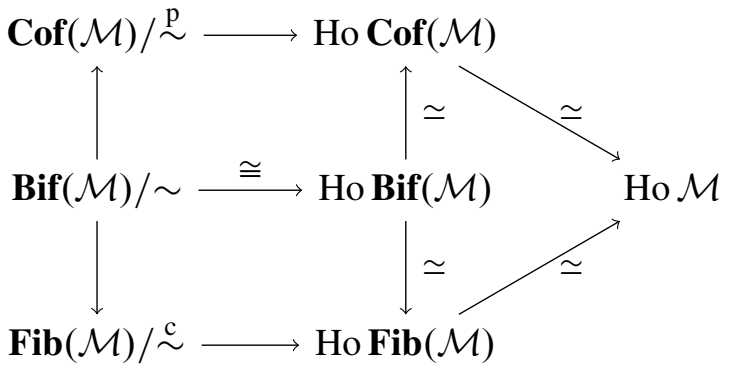

In this note, we treat the question whether the functors $\operatorname{Fib}(\mathcal{M}) / \stackrel{\mathrm{c}}{\sim} \rightarrow \operatorname{Ho} \operatorname{Fib}(\mathcal{M})$ and $\operatorname{Cof}(\mathcal{M}) / \stackrel{p}{\sim} \rightarrow \operatorname{Ho} \operatorname{Cof}(\mathcal{M})$ are faithful. By duality, it suffices to consider the first functor.

\section{The model category $\bmod (\mathbb{Z} / 4)$}

The category $\bmod (\mathbb{Z} / 4)$ of finitely generated modules over $\mathbb{Z} / 4$ is a Frobenius category (with respect to all short exact sequences), that is, there are enough projective and injective objects in $\bmod (\mathbb{Z} / 4)$ and, moreover, these objects coincide (we call such objects bijective). Therefore $\bmod (\mathbb{Z} / 4)$ carries a canonical model category structure (cf Hovey [4, Section 2.2]): The cofibrations are the monomorphisms and the fibrations are the epimorphisms in $\bmod (\mathbb{Z} / 4)$. Every object in $\bmod (\mathbb{Z} / 4)$ is bifibrant, and the weak equivalences are precisely the homotopy equivalences, where parallel morphisms $f$ and $g$ are homotopic if $g-f$ factors over a bijective object in $\bmod (\mathbb{Z} / 4)$. That is, 
the weak equivalences in $\bmod (\mathbb{Z} / 4)$ are the stable isomorphisms and the homotopy category of $\bmod (\mathbb{Z} / 4)$ is isomorphic to the stable category [2, Chapter I, Section 2.2] of $\bmod (\mathbb{Z} / 4)$.

We remark that every object in $\bmod (\mathbb{Z} / 4)$ is isomorphic to $(\mathbb{Z} / 4)^{\oplus k} \oplus(\mathbb{Z} / 2)^{\oplus l}$ for some $k, l \in \mathbb{N}_{0}$, and every bijective object is isomorphic to $(\mathbb{Z} / 4)^{\oplus k}$ for some $k \in \mathbb{N}_{0}$.

\section{Faithfulness of the functor $\operatorname{Fib}(\mathcal{M}) / \stackrel{\mathfrak{c}}{\sim} \rightarrow \operatorname{Ho} \operatorname{Fib}(\mathcal{M})$}

We give a sufficient criterion for the functor under consideration to be faithful.

Proposition If the model category $\mathcal{M}$ is left proper and if $w \amalg w$ is a weak equivalence for every weak equivalence $w$ in $\mathcal{M}$, then $\stackrel{c}{\sim}$ is a congruence on $\operatorname{Fib}(\mathcal{M})$ and the canonical functor $\operatorname{Fib}(\mathcal{M}) / \stackrel{\mathrm{c}}{\sim} \rightarrow \operatorname{Ho} \operatorname{Fib}(\mathcal{M})$ is faithful.

Proof We suppose given fibrant objects $X$ and $Y$ and morphisms $f, g: X \rightarrow Y$ with $\Gamma f=\Gamma g$ in $\operatorname{Ho} \operatorname{Fib}(\mathcal{M})$. By [1, Theorem 1(ii)], there exists a weak equivalence $w: X^{\prime} \rightarrow X$ such that $w f \stackrel{\mathrm{p}}{\sim} w g$. It follows that $w f \stackrel{\mathrm{c}}{\sim} w g$ by [5, Chapter I, Section 1, dual of Lemma 5], that is, there exists a cylinder $Z^{\prime}$ for $X^{\prime}$ and a cylinder homotopy $H^{\prime}: Z^{\prime} \rightarrow Y$ from $w f$ to $w g$. We let

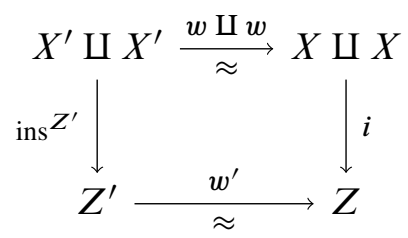

be a pushout of $w \amalg w$ along ins $Z^{\prime}$. By assumption, $w \amalg w$ and $w^{\prime}$ are weak equivalences. Since

$$
(w \amalg w)\left(\begin{array}{l}
1 \\
1
\end{array}\right)=\text { ins }^{Z^{\prime}} \mathrm{s}^{Z^{\prime}} w,
$$

there exists a unique morphism $s: Z \rightarrow X$ with

$$
\left(\begin{array}{l}
1 \\
1
\end{array}\right)=i s \text { and } \mathrm{s}^{Z^{\prime}} w=w^{\prime} s .
$$

Then $s$ is a weak equivalence since $\mathrm{s}^{Z^{\prime}}, w$ and $w^{\prime}$ are weak equivalences and therefore $Z$ becomes a cylinder for $X$ with ins ${ }^{Z}:=i$ and $\mathrm{s}^{Z}:=s$. Moreover,

$$
(w \amalg w)\left(\begin{array}{l}
f \\
g
\end{array}\right)=\operatorname{ins}^{Z^{\prime}} H^{\prime}
$$

implies that there exists a unique morphism $H: Z \rightarrow Y$ with

$$
\left(\begin{array}{c}
f \\
g
\end{array}\right)=\operatorname{ins}^{Z} H \quad \text { and } \quad H^{\prime}=w^{\prime} H .
$$


So in particular $f \stackrel{\mathrm{c}}{\sim} g$.

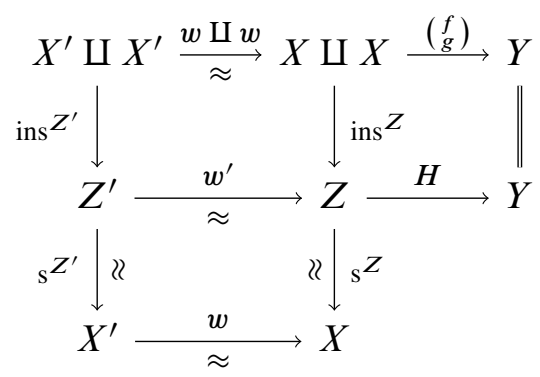

Altogether, we have shown that morphisms in $\operatorname{Fib}(\mathcal{M})$ represent the same morphism in $\operatorname{Ho} \mathbf{F i b}(\mathcal{M})$ if and only if they are cylinder homotopic. In particular, $\stackrel{c}{\sim}$ is a congruence on $\operatorname{Fib}(\mathcal{M})$.

The following counterexample shows that the canonical functor

$$
\operatorname{Fib}(\mathcal{M}) / \stackrel{\mathfrak{c}}{\sim} \rightarrow \operatorname{Ho} \operatorname{Fib}(\mathcal{M})
$$

is not faithful in general.

Example We consider the category $(\mathbb{Z} / 4 \downarrow \bmod (\mathbb{Z} / 4))$ of finitely generated $\mathbb{Z} / 4$ modules under $\mathbb{Z} / 4$ with the model category structure inherited from $\bmod (\mathbb{Z} / 4)$; see Section 2 . All objects in $(\mathbb{Z} / 4 \downarrow \bmod (\mathbb{Z} / 4))$ are fibrant since all objects in $\bmod (\mathbb{Z} / 4)$ are fibrant.

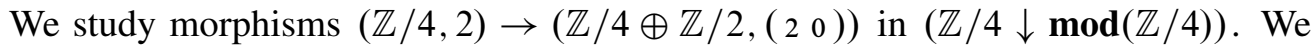
let $(Z, t)$ be a cylinder of $(\mathbb{Z} / 4,2)$ and we let $H:(Z, t) \rightarrow(\mathbb{Z} / 4 \oplus \mathbb{Z} / 2,(20))$ be a cylinder homotopy (from $\operatorname{ins}_{0} H$ to ins $_{1} H$ ). Then we have a weak equivalence $(Z, t) \rightarrow(\mathbb{Z} / 4,2)$ in $(\mathbb{Z} / 4 \downarrow \bmod (\mathbb{Z} / 4))$ and hence a weak equivalence $Z \rightarrow \mathbb{Z} / 4$ in $\bmod (\mathbb{Z} / 4)$. Thus $Z$ is bijective and therefore we may assume that $Z=(\mathbb{Z} / 4)^{\oplus k}$. Since ins ${ }_{0}$ and ins ${ }_{1}$ are morphisms from $(\mathbb{Z} / 4,2)$ to $(Z, t)$, we have 2 ins $_{0}=t=2$ ins $_{1}$ and hence ins $_{0} \equiv_{2}$ ins $_{1}$ as morphisms from $\mathbb{Z} / 4$ to $Z$. But this implies that the second components of $\operatorname{ins}_{0} H$ and ins $_{1} H$ are the same. In other words, we have shown that cylinder homotopic morphisms from $(\mathbb{Z} / 4,2)$ to $(\mathbb{Z} / 4 \oplus \mathbb{Z} / 2,(20))$ coincide in the second component. It follows that the morphisms $\left(\begin{array}{ll}1 & 0\end{array}\right):(\mathbb{Z} / 4,2) \rightarrow$ $\left(\mathbb{Z} / 4 \oplus \mathbb{Z} / 2,\left(\begin{array}{ll}2 & 0\end{array}\right)\right)$ and $\left(\begin{array}{ll}1 & 1\end{array}\right):(\mathbb{Z} / 4,2) \rightarrow\left(\mathbb{Z} / 4 \oplus \mathbb{Z} / 2,\left(\begin{array}{lll}2 & 0\end{array}\right)\right)$ in $(\mathbb{Z} / 4 \downarrow \bmod (\mathbb{Z} / 4))$ represent different morphisms in the quotient category $\operatorname{Fib}((\mathbb{Z} / 4 \downarrow \bmod (\mathbb{Z} / 4))) / \stackrel{\mathrm{c}}{\sim}$.

On the other hand, since $\mathbb{Z} / 4$ is bijective, the morphism $2: \mathbb{Z} / 4 \rightarrow \mathbb{Z} / 4$ is a weak equivalence in $\bmod (\mathbb{Z} / 4)$, and therefore $2:(\mathbb{Z} / 4,1) \rightarrow(\mathbb{Z} / 4,2)$ is a weak equivalence in $(\mathbb{Z} / 4 \downarrow \bmod (\mathbb{Z} / 4))$. But $2\left(\begin{array}{ll}1 & 0\end{array}\right)=2\left(\begin{array}{ll}1 & 1\end{array}\right)$ as morphisms from $(\mathbb{Z} / 4,1)$ to

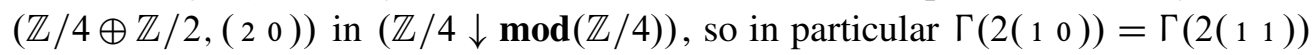
and hence $\Gamma\left(\begin{array}{ll}1 & 0\end{array}\right)=\Gamma\left(\begin{array}{ll}1 & 1\end{array}\right)$. 


\section{References}

[1] KS Brown, Abstract homotopy theory and generalized sheaf cohomology, Trans. Amer. Math. Soc. 186 (1974) 419-458 MR0341469

[2] D Happel, Triangulated categories in the representation theory of finite-dimensional algebras, London Math. Society Lecture Note Ser. 119, Cambridge Univ. Press (1988) MR935124

[3] PS Hirschhorn, Model categories and their localizations, Math. Surveys and Monogr. 99, Amer. Math. Soc. (2003) MR1944041

[4] M Hovey, Model categories, Math. Surveys and Monogr. 63, Amer. Math. Soc. (1999) MR1650134

[5] D G Quillen, Homotopical algebra, Lecture Notes in Math. 43, Springer, Berlin (1967) MR0223432

Department of Mathematics, University of Notre Dame Notre Dame, IN 46556, USA

86 Cedar $S t$

Lexington, MA 02421, USA

Lehrstuhl D für Mathematik, RWTH Aachen University

Templergraben 64, D-52062 Aachen, Germany

dwyer.1@nd.edu, andrei@alum.mit.edu, sebastian.thomas@math.rwth-aachen.de

http://www.nd.edu/ wgd/, http://www .math.rwth-aachen.de/ Sebastian. Thomas/

Received: 6 October 2009 